\title{
Effects of contextual constraint on eye movements in reading: A further examination
}

\author{
KEITH RAYNER and ARNOLD D. WELL \\ University of Massachusetts, Amherst, Massachusetts
}

\begin{abstract}
The effect of contextual constraint on eye movements in reading was examined by asking subjects to read sentences that contained a target word that varied in contextual constraint; high-, medium-, or low-constraint target words were used. Subjects fixated low-constraint target words longer than they did either high- or medium-constraint target words. In addition, they skipped high-constraint words more than they did either medium- or low-constraint target words. The results further confirm that contextual constraint has a strong influence on eye movements during reading.
\end{abstract}

A number of studies have demonstrated that context influences the processing of words (e.g., Becker, 1985; Ehrlich \& Rayner, 1981; Fischler \& Bloom, 1979; Stanovich \& West, 1983). Most of these studies have employed either naming (Stanovich \& West, 1979, 1981; West \& Stanovich, 1982) or lexical decision tasks (Fischler \& Bloom, 1979; Schuberth \& Eimas, 1977). Typically, a sentence fragment is presented and then subjects respond, by making a naming or lexical decision response, to a target word (which is presented immediately after the context). The contexts are either congruent or incongruent with the target word. The general finding from these studies is that subjects respond faster to congruent than to incongruent target words.

In addition to the naming and lexical decision studies, there have also been a number of eye-movement studies which have examined the influence of context when reading is not disrupted by making a response to an isolated target word. In other words, from the subjects' point of view, there is nothing special about the target word inasmuch as it is not distinguished in any way from other words in the text (as is the case in the naming and lexical decision experiments where the target word is presented in isolation following the context). In the eye-movement studies, it has generally been found that (1) highly constrained target words are skipped (i.e., not directly fixated) more frequently than unconstrained words (Altarriba, Kroll, Sholl, \& Rayner, 1996; Balota, Pollatsek, \& Rayner, 1985; Ehrlich \& Rayner, 1981); (2) more regressions are made to unconstrained than to constrained words (Ehrlich \& Rayner, 1981; Inhoff, 1984); and (3) when target words are

This research was supported by Grant DBS91-21375 from the National Science Foundation and Grant HD26765 from the National Institutes of Health. The first author was also supported by a Research Scientist Award from the National Institute of Mental Health (MH01255). We thank Lori Rogg for her assistance in collecting and analyzing the data and Charles Clifton, Jukka Hyona, Betty Ann Levy, Randi Martin, and Marilyn Smith for their helpful comments on an earlier version of this article. Requests for reprints should be sent to K. Rayner, Department of Psychology, University of Massachusetts, Amherst, MA 01003 (e-mail: rayner@psych.umass.edu). fixated, fixation time is shorter on constrained than on unconstrained words (Altarriba et al., 1996; Balota et al., 1985; Ehrlich \& Rayner, 1981; Inhoff, 1984; Schustack, Ehrlich, \& Rayner, 1987; Zola, 1984). With respect to the third finding, first-fixation duration (the duration of the first fixation on the target word independent of the number of fixations), gaze duration (the sum of all fixations on a word prior to movement to another word), and the total fixation time (the sum of all fixations on a word, including regressions) are all shorter for constrained words in comparison with unconstrained words.

In the eye-movement experiments, as in the naming and lexical decision experiments, sentential constraint has typically been determined via a cloze task in which subjects (who did not participate in the eye-movement study) are presented with a sentence fragment (or paragraph) up to the target word and instructed to write down the next word. Target words that subjects produce a high percentage of the time (typically over $60 \%$ of the time, and often as high as $80 \%$ or $90 \%$ of the time) are considered to be highly constrained; target words that subjects correctly write down less than $10 \%$ of the time are considered to be relatively unconstrained. High- and low-constraint target words therefore are determined in advance of the experiment.

Whereas all of the eye-movement studies mentioned above found effects of constraint on either word skipping or fixation time, a recent study reported by Hyona (1993) found no effects of constraint. Hyona's experiment differs from the others in that the sentence completions for the target words (also determined in advance of the experiment) were somewhat different from the typical experiment. In particular, the high-constraint target words were correctly identified on the cloze task $65 \%$ of the time and the low-constraint target words were identified $32 \%$ of the time. Thus, the difference in predictability between the high- and low-constraint target words was not as great as it was in the other studies. Hyona found that first- fixation duration and gaze duration did not differ for the two types of target words, and nor did the probability of skipping the target word. However, there was a suggestion that 
total fixation time on the target word was longer for lowconstraint than for high-constraint target words, and there were more regressions to the low-constraint target word than to the high-constraint target word (a finding also reported by Ehrlich \& Rayner, 1981, and by Inhoff, 1984).

As Hyona (1993) noted, his study was most similar to that of Ehrlich and Rayner (1981). He attributed the difference in results between his study and their's to the variation in predictability between the constrained and unconstrained word in the two studies; in Ehrlich and Rayner's first experiment, the probability of a correct completion in the cloze task was $93 \%$ for high-constraint targets and $15 \%$ for low-constraint targets, whereas in their second experiment, the corresponding values were $60 \%$ and $0 \%$. Hyona concluded that "strong contextual manipulations are needed to produce a reliable context effect for the firstpass reading of a word" (p. 300).

Although Hyona's conclusion may well be correct, our view is that additional data are necessary before such a conclusion can be reached. In Hyona's study, the average word length of the target word was 8.8 characters (range = 6-13 characters), whereas in the Ehrlich and Rayner study, all target words were 5 characters long. Given that, independent of the degree of contextual constraint, readers are much less likely to skip long words ( 8 letters or more) than words that are 5-7 letters long (see Rayner \& McConkie, 1976; Rayner, Sereno, \& Raney, in press), the target words to be used should be shorter than those used by Hyona. In addition, data collected from the same subjects are needed when contextual constraint is varied over a larger range than has typically been the case. Indeed, this was the goal of the present experiment. In addition to providing data over a wider range of contextual constraint, the present results are important because a recent model of eye-movement control in reading (Reichle, Pollatsek, Fisher, \& Rayner, 1996) relies heavily on contextual constraint in simulating the reading process. If contextual constraint has an influence only when words are highly predictable, such a finding would be problematical for the Reichle et al. model. Therefore, in the present experiment, subjects read sentences in which target words varied over a larger range of predictability values (as measured by a cloze task) than has typically been the case. Additionally, the target words' lengths were shorter than in Hyona's experiment.

\section{METHOD}

\section{Subjects}

Eighteen members of the University of Massachusetts community participated in the experiment. They all had normal uncorrected vision and were native speakers of English.

\section{Apparatus}

Eye movements were recorded by a Fourward Technologies Dual Purkinje Eyetracker which has a resolution of less than $10^{\prime}$ of arc. The eyetracker was interfaced with an Epson Equity III computer. The position of the eye was sampled every millisecond, and the computer stored data on the duration and location of each fixation for later analysis. The computer was also interfaced with a Sony Trinitron display on which the sentences were presented. The display was $80 \mathrm{~cm}$ from the subject's eye
}

and three characters equaled $1^{\circ}$ of visual angle. Viewing was binocular, but eye movements were recorded from the subject's right eye. A bitebar was used to eliminate head movements in the experiment.

\section{Materials}

Thirty-six target sentences were adopted from norms provided by Schwanenflugel (1986). Her procedure was to allow subjects to list up to, but not more than, three completions for each target sentence. For example, in the target sentence "The woman took the warm cake out of the ," the word "oven" was produced $93 \%$ of the time, "stove" was produced $33 \%$ of the time, and "pantry" was produced $3 \%$ of the time. Thus, each of the target sentences provides a range of responses from the most frequent response to the least frequent response. Using these norms, we selected sentences so that there was an equal number of highconstraint target words (mean sentence completion $=86 \%$ of the time, range $=73 \%-100 \%$ ), medium-constraint words (mean sentence completion $=41 \%$ of the time, range $=13 \%-68 \%$, and low-constraint target words (mean sentence completion $=4 \%$, range $=3 \%-8 \%$ ). Twelve sentences contained either a high-constraint or a medium-constraint target, 12 contained either a medium- or low-constraint target, and 12 contained either a high- or a low-constraint target. Counterbalancing procedures ensured that each sentence (with each of the target words) was read equaly often. In selecting pairs of target words for each sentence from the Schwanenflugel norms, we matched as closely as possible on word length and word frequency; the average target word length was $6.0,5.9$, and 6.2 letters for the high-, medium-, and low-constraint target words, respectively; and the average word frequency according to the Francis and Kučera (1982) norms was 55, 60, and 61 per million. The appendix lists the sentences and target words (with their appropriate sentence-completion scores).

\section{Procedure}

When a subject arrived for the experiment, the bite bar was prepared and the eyetracking system was calibrated. The calibration period usually lasted less than $5 \mathrm{~min}$. After the calibration was completed, subjects were told that they would be asked to read a number of sentences, and that the purpose of the experiment was to determine what people looked at as they read. They were also told that they would periodically be asked to answer comprehension questions about the sentences. On approximately one-third of the trials, the experimenter asked the subject to release the bite bar and answer a question. The subjects had no difficulty answering these questions; the questions were simple true-false questions, and the subjects were correct about $90 \%$ of the time. Following the initial calibration, the subjects read six practice sentences before reading 36 experimental sentences. The experimental sentences were embedded in 108 filler sentences.

\section{RESULTS}

A number of different eye-movement measures were examined with respect to the target word. Specifically, the measures were: (1) first-fixation duration (the duration of the first fixation on the word independent of the number of fixations on the word), (2) gaze duration (the sum of all fixations on a word prior to moving to another word), (3) total fixation time (the sum of all fixations on a word, including regressions), and (4) the probability of fixating on the target word.

The region used for computing the probability of fixating the target word was the letters in the target word and the space preceding the target word. However, a broader region was used for computing the various fixation-time measures if readers did not directly fixate the target word. ${ }^{1}$ Specifically, the region used for computing first fixation and gaze duration was the target word, the space prior to it, and the last three characters of the word preceding it. The rationale for doing this was that readers often iden- 
tify short words when they are fixated just to the left of the word (see Rayner \& Pollatsek, 1989). Approximately $4 \%$ of the data were eliminated either because of track losses or because the fixations were shorter than $100 \mathrm{msec}$; see Morrison (1984) and Rayner, Sereno, Morris, Schmauder, and Clifton (1989) for justifications for eliminating fixations shorter than $100 \mathrm{msec}$.

\section{Fixation Time}

As shown in Table 1, the three fixation-time measures showed exactly the same pattern: the high- and mediumconstraint target words did not differ from each other, but the low-constraint target words yielded longer fixation times than the high-or medium-constraint target words. An analysis of variance (ANOVA) on each of the three sets of data, using subjects $(F 1)$ and items $(F 2)$ as random effects, revealed that for first-fixation duration the constraint effect was only marginally significant by subjects $\left[F 1(2,34)=2.70, M S_{\mathrm{e}}=944, p<.10\right]$ and nonsignificant by items $(F 2<1)$, while for the other two measures, the constraint effect was significant: for gaze duration $\left[F 1(2,34)=4.64, M S_{\mathrm{e}}=1,016, p<.05 ; F 2(2,46)=\right.$ $\left.3.63, M S_{\mathrm{e}}=1,344, p<.05\right]$ and for total time ${ }^{2}[F 1(2,34)=$ $13.11, M S_{\mathrm{e}}=1,826, p<.001 ; F 2(2,46)=3.86, M S_{\mathrm{e}}=$ $9,164, p<.05]$. Post hoc Bonferroni $t$ tests further revealed no difference between the high- and medium-constraint conditions, while each of these conditions differed from the low-constraint condition ( $p s<.05$ ).

\section{Fixation Probability}

The probability of a first-pass fixation on the target word showed a pattern different from that of the fixation time measures. Specifically, readers were more likely to skip over the high-constraint target word than either the medium- or the low-constraint target word. An ANOVA on the data yielded an overall effect of constraint $[F 1(2,34)=$ $9.1, M S_{\mathrm{e}}=88, p<.001 ; F 2(2,46)=6.29, M S_{\mathrm{e}}=170$, $p<.01]$. Post hoc Bonferroni $t$ tests further revealed no difference between the medium- and low-constraint conditions, while each differed from the high-constraint condition $(p s<.05)$.

\section{Regression Analyses}

To gain additional information and because word length and word frequency were not perfectly matched (since we used words from the Schwanenflugel norms), correlations were calculated and regression analyses were performed on the data. The advantage of a regression analysis of the sort we did is that it takes account of the quantitative pre-

Table 1

Fixation Time Measures (in Milliseconds) and Fixation Probability on Target Word as a Function of Constraint

\begin{tabular}{lcccc}
\hline & \multicolumn{3}{c}{ Fixation Time } & \\
\cline { 2 - 5 } Constraint & $\begin{array}{c}\text { First } \\
\text { Fixation }\end{array}$ & $\begin{array}{c}\text { Gaze } \\
\text { Duration }\end{array}$ & $\begin{array}{c}\text { Total } \\
\text { Time }\end{array}$ & $\begin{array}{c}\text { Fixation } \\
\text { Probability }\end{array}$ \\
\hline High & 239 & 261 & 294 & .78 \\
Medium & 240 & 261 & 301 & .88 \\
Low & 250 & 281 & 360 & .90 \\
\hline
\end{tabular}

dictability values associated with the high-, medium-, and low-constraint categories, and also accounts for the fact that there wasn't perfect matching of word length and frequency. Lorch and Myers (1990) have demonstrated that for within-subject designs, it is inappropriate to collapse across subjects because the appropriate error term for a test of any treatment source of variance in the within-subjects section of the analysis is the corresponding subject $X$ treatment interaction. Thus, separate regression equations were calculated for each subject, using the predictor variables of word length, word frequency, and predictability (i.e., percentage sentence completion), and a $t$ test was performed on the partial regression coefficients for each predictor. The correlation between length and frequency was .01 ; that between predictability and frequency was -.05 . The only correlation significant with a Bonferroni pairwise correction was that of length with predictability $(p<.01)$. Yet even this correlation was minimal $(r=-.12)$, thus indicating that the contributions of the predictor variables were largely independent.

For the dependent variables, first fixation, gaze duration, and total time, ordinary least squares regressions with predictors of length, predictability, and frequency were performed. For the probability of fixating the target word, because whether or not a subject fixated a target word is a dichotomous variable, a logistic regression with the same predictors was performed. The resulting coefficients are shown in Table 2.

Tests of the partial regression coefficients revealed that word length was not a useful predictor of first-fixation duration, gaze duration, or total fixation time when the effects of predictability and frequency were partialed out $(t \mathrm{~s}<1)$. On the other hand, for the first-fixation-duration regression equation, frequency was significant $[t(17)=2.24$, $p<.05]$, whereas for the gaze-duration regression equation, predictability was marginally significant $[t(17)=$ $2.01, p<.06]$ and frequency was significant $[t(17)=$ $2.98, p<.01]$; for the total-time regression equations, both were significant $[t(17)=4.69, p<.001$, for predictability; $t(17)=2.69, p<.05$, for frequency]. There was also evidence for a quadratic component to the relation between total fixation time and predictability, consistent with the finding that the effect of medium constraint was more similar to the effect of high constraint than to that of low constraint. The partial regression coefficient of (predictability) (see note 2) was significant in an equation that also contained frequency, length, and predictability $[t(17)=2.63, p<.05]$. The same general pattern of results was present for first fixation and gaze duration, but the quadratic component did not reach significance in these measures. For probability of fixating the target word, predictability $[t(17)=3.24, p<.001]$ and length $[t(17)=2.51, p<.05]$ were both significant predictors, while frequency was not $[t(17)=1.59, p>.10]$.

\section{DISCUSSION}

The results of the present study clearly confirm prior findings (A1tarriba et al., 1996; Balota et al., 1985; Ehrlich \& Rayner, 1981) that 
Table 2

Mean Coefficient for Each Predictor and Dependent Measure

\begin{tabular}{lccc}
\hline & Length & Predictability & Frequency \\
\hline First fixation & .250 & -.110 & -.094 \\
Gaze duration & .239 & -.295 & -.197 \\
Total time & 1.536 & -.853 & -.256 \\
Fixation probability & 1.465 & -.032 & -.005 \\
\hline
\end{tabular}

contextual constraint influences eye-movement behavior during reading. Specifically, our results demonstrate that highly constrained target words are fixated for less time and skipped more frequently than unconstrained words. Our results are also consistent with prior findings which have demonstrated that (1) word frequency influences fixation time on a word (see also Altarriba et al., 1996; Inhoff \& Rayner, 1986; Rayner \& Duffy, 1986; Rayner \& Raney, 1996; Rayner et al., in press) and (2) word length influences the probability of fixating a word (see Rayner \& McConkie, 1976; Rayner et al., in press). It is also interesting to note that the regression analyses suggest that for the relatively restricted range of word lengths used in our study, word length had little effect on either first-fixation duration or gaze duration, whereas frequency and predictability did. On the other hand, word length and predictability influenced word skipping, whereas frequency did not.

More importantly, the present results clarify the apparent discrepancy between results reported by Ehrlich and Rayner (1981) and by Hyona (1993) in dealing with fixation times and word skipping as a function of contextual constraint. Whereas Ehrlich and Rayner found that readers fixated words that were highly constrained by context for less time than they did words that were not constrained by preceding context, Hyona found no difference. As noted in the introduction to this article, Hyona's target words were closer together in terms of predictability scores (.65 vs. 32 on a cloze task) than were Ehrlich and Rayner's target words (.93 vs. 15 close predictability in Experiment 1 and .60 vs. .00 in Experiment 2). In the present study, we found that highly constrained target words (average cloze predictability of .86) and moderately constrained target words (average predictability of .41) were fixated for less time than were unconstrained target words (average predictability of .04). Thus, with respect to fixation times, it would appear that Hyona's suggestion that strong contextual manipulations are needed to influence the initial or first-pass reading of words is not correct. Rather, it seems that, as far as fixation times of words are concerned, words that are unconstrained by context are fixated longer than words that are moderately to highly constrained. Given that Hyona did not have a condition similar to our unconstrained condition, no effect of constraint on fixation times was obtained in his study.

With respect to the probability of skipping a word, Ehrlich and Rayner reported that highly constrained words were skipped more frequently than unconstrained words, whereas Hyona again found no difference as a function of constraint. In the present study, the word-skipping results were consistent with Hyona's suggestion: highly constrained words were skipped more frequently than either moderately or unconstrained words. Thus, it would appear that Hyona was partially correct when he suggested that strong contextual manipulations were needed to influence eye-movement behavior on first-pass reading. Strong constraint is necessary to influence word skipping but not first-pass fixation times. Because Hyona did not have a high-constraint condition (comparable to the one we used), it is not surprising that he obtained no effect of word skipping, since his study included conditions that are most similar to our medium-constraint condition.

The difference in the pattern of results for fixation time versus word skipping in our data can best be accounted for by the notion that different mechanisms are involved in the decisions about when and where to move the eyes (see Rayner \& McConkie, 1976; Rayner \& Pollatsek, 1981). According to this view, the when decision (which results in fixationtime effects) is driven by the cognitive processes associated with understanding the fixated word, while the where decision (which results in word skipping) is driven largely by low-level visual features, such as the length of the upcoming word. Although the decision about where to fixate next is primarily determined by low-tevel information, it is also known that contextual constraint can influence the effectiveness of parafoveal processing. Given that more parafoveal information is obtained from constrained words than from unconstrained words (Balota et al., 1985), the probability that such a word in parafoveal vision will be identified (and hence skipped) is higher than it would be if the same word was not constrained by the context. Thus, if we consider target words that are between 5 and 7 letters long, a variable such as word frequency will affect fixation time but have little effect on word skipping, while a variable such as word length will affect word skipping but have less of an effect on fixation times (see Rayner et al., in press). Predictability of a word for the amount of contextual constraint for that word), on the other hand, will affect both fixation time and word skipping.

In general, all of our results are consistent with a model of eyemovement control in reading (Morrison, 1984; Rayner et al., in press; Reichle et al., 1996) in which moment-to-moment processing influences the decision of when to move the eyes. For example, Reichle et al. have described a moment-to-moment processing model that accurately simulates fixation times and word skipping. In that model, the ease of lexical access of fixated words determines when the eyes move and both word frequency and contextual constraint (predictability) modulate the fixation time on a word, with more frequent words and more constrained words receiving shorter fixations than infrequent or unconstrained words. Words that are highly constrained by the context are also skipped more frequently than unconstrained words in the model. These patterns, of course, are exactly what was observed in the data reported here.

\section{REFERENCES}

Altarriba, J., Kroll, J. F., Sholl, A., \& Rayner, K. (1996). The influence of lexical and conceptual constraints on reading mixedlanguage sentences: Evidence from eye fixations and naming times. Memory \& Cognition, 24, 477-492.

Balota, D. A., Pollatsek, A., \& Rayner, K. (1985). The interaction of contextual constraints and parafoveal visual information in reading. Cognitive Psychology, 17, 364-390.

BECKER, C. A. (1985). What do we really know about semantic context effects during reading? In D. Besner, T. G. Waller, \& G. E. MacKinnon (Eds.), Reading research: Advances in theory and practice (Vol, 5). New York: Academic Press.

EHRlich, S. F., \& RAYNER, K. (1981). Contextual effects on word perception and eye movements during reading. Journal of Verbal Learning \& Verbal Behavior, 20, 64 I-655.

FisCHLER, I., \& BLOOM, P. (1979). Automatic and attentional processes in the effects of sentence context on word recognition. Journal of Verbal Learning \& Verbal Behavior, 18, 1-20.

FRANCIS, W. N., \& KuČERA, H. (1982). Frequency analysis of English usage: Lexicon and grammar. Boston: Houghton Mifflin.

HYONA, J. (1993). Effects of thematic and lexical priming on readers' eye movements. Scandinavian Journal of Psychology, 34, 293-304.

INHOFF, A. W. (1984). Two stages of word processing during eye fixations in the reading of prose. Journal of Verbal Learning \& Verbal Behavior, 23, 612-624

INHOFF, A. W., \& RAYNER, K. (1986). Parafoveal word processing during eye fixations in reading: Effects of word frequency. Perception \& Psychophysics, 40, 431-439.

LORCH, R. F., \& MYERS, J. L. (1990). Regression analyses of repeated measures data in cognitive research. Journal of Experimental Psychology: Learning, Memory, \& Cognition, 16, 149-157.

MoRRISON, R. E. (1984). Manipulations of stimulus onset delay in reading: Evidence for parallel programming of saccades. Journal of Experimental Psychology: Human Perception \& Performance, 10, 667-682.

RaYNER, K., \& DufFY, S. A. (1986). Lexical complexity and fixation times in reading: Effects of word frequency, verb complexity, and lexical ambiguity. Memory \& Cognition, 14, 191-201.

RAYNER, K., \& MCCONKIE, G. W. (1976). What guides a reader's eye movements? Vision Research, 16, 829-837.

Rayner, K., \& Pollatsek, A. (1981). Eye movement control in reading: Evidence for direct control. Quarterly Journal of Experimental Psychology, 33A, 351-373.

RAYNER, K., \& RANEY, G. E. (1996). Eye movement control in reading and visual search: Effects of word frequency. Psychonomic Bulletin \& Review, 3, 245-248. 
Rayner, K., Sereno, S. C., Morris, R. K., Schmauder, A. R., \& CLIFTON, C. (1989). Eye movements and on-line language comprehension processes. Language \& Cognitive Processes, 4, 1-50.

RAYNER, K., SERENo, S. C., \& RANEy, G. E. (in press). Eye movement control in reading: A comparison of two types of models. Journal of Experimental Psychology: Human Perception \& Performance.

Reichle, E., Pollatsek, A., Fisher, D. L., \& Rayner, K. (1996). Toward a model of eye-movement control in reading. Manuscript submitted for publication.

Schuberth, R. E., \& Eimas, P. D. (1977). Effects of context on the classification of words and nonwords. Journal of Experimental Psychology: Human Perception \& Performance, 3, 27-36.

SCHUSTACK, M. W., EhrLICH, S. F., \& RAYNER, K. (1987). Local and global sources of contextual facilitation in reading. Journal of Verbal Learning \& Verbal Behavior, 26, 255-276.

SCHWANENflugel, P. J. (1986). Completion norms for final words of sentences using a multiple production measure. Behavior Research Methods, Instruments, \& Computers, 18, 363-371.

StANOVICH, K. E., \& WEST, R. F. (1979). Mechanisms of sentence context effects in reading: Automatic activation and conscious attention. Memory \& Cognition, 7, 77-85.

StanOVich, K. E., \& WeST, R. F. (1981). The effect of sentence context on ongoing word recognition: Tests of a two-process theory. Journal of Experimental Psychology: Human Perception \& Performance, 7, 658-672.
Stanovich, K. E., \& West, R. F. (1983). On priming by a sentence context. Journal of Experimental Psychology: General, 112, 1-36.

West, R. F., \& StaNovich, K. E. (1982). Source of inhibition in experiments on the effect of sentence context on word recognition. Journal of Experimental Psychology: Learning, Memory, \& Cognition, 8 , 385-399.

ZoLA, D. (1984). Redundancy and word perception during reading. Perception \& Psychophysics, 36, 277-284.

\section{NOTES}

1. Exactly the same data pattern and results were obtained when the only data we included were those in which the subject fixated the target word directly.

2. Since the total time measure includes-regressions to the target word from a later point in the sentence, the fact that the difference between the low-constraint condition and the other two conditions is more pronounced in the total time measure suggests that more regressions were made in the low-constraint condition than in the other two conditions. Indeed, consistent with prior reports, subjects regressed more frequently in the low-constraint condition (27\%) than in the other two conditions (roughly $16 \%$ ). However, there was a considerable amount of variability among the subjects in terms of the frequency of regressions, and the effect was not significant.

\section{APPENDIX}

\section{High-Medium Sentences}

1. The woman took the warm cake out of the __ so that she could frost it. oven $=.98 ;$ stove $=.23$

2. He campaigned so he would win the in his home state.

3. My uncle gave my mother a big

4. He mailed the letter without a when he saw her.

5. Harriet sang while my brother played the for my birthday.

6. While away, James sent home a to keep in touch.

7. The landlord was faced with a strike by the of the building.

8. Our new green car blocked the

9. The old man and woman married for

10. The cup was placed on the with great care. to the neighbor's house.

11. To keep her hands soft, Carol put on the ___ when she gardened.

12. Some of the ashes dropped on the ___ to the host's dismay.

\section{Medium-Low Sentences}

13. Calm seas are always good for 14. They were startled by the sudden

15. New clues led to the criminal's

16. Helen reached up to dust the

17. The girl crept slowly toward the

18. Wild animals can easily detect on hot summer days. from the next room years after the crime. above the fireplace. without anyone hearing her. as a matter of survival

19. The businessman brought his equipment to play

20. The breeze lifted the up from the ground. with his son.

21 . She was the only one to see the

22. Mary collapsed during the and was later questioned.

23 . He jumped with pleasure when he saw the due to the extreme heat.

24 . His failure was due greatly to a lack of on the table. in that area.

\section{High-Low Sentences}

25. The postman opened the package to inspect its before sending it.

26. To keep the animals out of the garden, he put up a to block it off

27 . The hikers slowly climbed up the to get a better view.

28. Jill looked back through the open election $=.98 ;$ primary $=.20$

kiss $=.95$; hug $=.63$

stamp $=1.00 ;$ address $=.40$

piano $=.88 ;$ flute $=.38$

letter $=.88 ;$ package $=.28$

tenants $=.83 ;$ workers $=.45$

driveway $=.83 ;$ entrance $=.15$

love $=.80 ;$ money $=.65$

table $=.80 ;$ saucer $=.53$

lotion $=.75 ;$ gloves $=.58$

floor $=.73 ;$ carpet $=.50$

swimming $=.65$; floating $=.05$

noise $=.68 ;$ voice $=.03$

arrest $=.55 ;$ pardon $=.03$

shelf $=.53 ;$ ledge $=.05$

door $=.45 ;$ edge $=.05$

food $=.48 ;$ rain $=.03$

golf $=.35 ;$ ball $=.05$

leaves $=.38$; debris $=.03$

accident $=.28$; shooting $=.03$

race $=.25 ;$ hike $=.03$

present $=.18 ;$ picture $=.05$

knowledge $=.13 ;$ experience $=.03$

contents $=1.00 ;$ packing $=.05$

fence $=.95 ;$ hedge $=.03$

mountain $=.95 ;$ hillside $=.03$

window $=.93 ;$ curtain $=.03$ 


\section{APPENDIX (Continued)}

29. He scraped the cold food from his

30. The man decided to shave his

31. The worker was criticized by his

32. The friends were not talking because

33. The woman died after a prolonged

34. The trial received a lot of

35. The difficult task was beyond his

36. The car in front suddenly changed before washing it.

before the operation. but only behind his back.
to everyone's dismay.

all the local papers and he became frustrated. and almost lost control. plate $=.90 ;$ spoon $=.05$

beard $=.83$; chest $=.08$

boss $=.85 ;$ help $=.03$

fight $=.85 ;$ scheme $=.03$

illness $=.78 ;$ surgery $=.05$

publicity $=.78$; headlines $=.03$

ability $=.75$; capacity $=.05$

direction $=.73$; position $=.03$

(Manuscript received October 5, 1995;

revision accepted for publication April 23, 1996.) 